\title{
Two 22q telomere deletions serendipitously detected by FISH
}

Kathrin S Precht, Christa M Lese, Rhonda P Spiro, Peter R Huttenlocher, Kathreen M Johnston, John Ca Baker, Susan L Christian, Kirk Kittikamron, David H Ledbetter

Department of Human Genetics, The University of Chicago, 924 E 57th Street, Chicago, IL 60637, USA

K S Precht

$C M$ Lese

R P Spiro

S L Christian

K Kittikamron

D H Ledbetter

Department of Pediatrics, The

University of Chicago,

Chicago, IL, USA

P R Huttenlocher

D H Ledbetter

Kaiser Permanente, San Francisco, CA, USA

K M Johnston

Kaiser Permanente, Oakland, CA, USA

J C Baker

Correspondence to: Dr Ledbetter.

Received 3 March 1998 Revised version accepted for publication 22 April 1998

\begin{abstract}
Cryptic telomere deletions have been proposed to be a significant cause of idiopathic mental retardation. We present two unrelated subjects, with normal $G$ banding analysis, in whom 22q telomere deletions were serendipitously detected at two different institutions using fluorescence in situ hybridisation (FISH). Both probands presented with several of the previously described features associated with 22q deletions, including hypotonia, developmental delay, and absence of speech. Our two cases increase the total number of reported $22 \mathrm{q}$ telomere deletions to 19 , the majority of which were identified by cytogenetic banding analysis. With the limited sensitivity of routine cytogenetic studies $(\sim 2-5 \mathrm{Mb})$, these two new cases suggest that the actual prevalence of $22 \mathrm{q}$ telomere deletions may be higher than currently documented. Of additional interest is the phenotypic overlap with Angelman syndrome (AS) as it raises the possibility of a 22q deletion in patients in whom AS has been ruled out. The use of telomeric probes as diagnostic reagents would be useful in determining an accurate prevalence of chromosome 22q deletions and could result in a significantly higher detection rate of subtelomeric rearrangements.

(F Med Genet 1998;35:939-942)
\end{abstract}

Keywords: del(22)(q13.3); absent speech; Angelman syndrome

Since the initial description of a potential partial monosomy 22q syndrome in 1985 by Watt et al, ${ }^{1}$ a total of 17 cases of monosomy 22q13.3 have been reported. Two of the deletions were the product of gross cytogenetic rearrangements, ${ }^{12} 11$ were cytogenetically apparent terminal deletions, ${ }^{3-8}$ and two additional cases were more subtle "suspected" deletions, later confirmed by FISH. ${ }^{9}$ Two submicroscopic deletions were detected by Southern blot analysis in a screening of a population of mentally retarded subjects. ${ }^{10}$

The patients reported to have $22 \mathrm{q} 13$ deletions share several clinical features. Many of the patients presented with developmental delay, particularly of motor milestones. In addition, most were hypotonic. Interestingly, in all reported cases, expressive language was either absent, severely delayed, or unascertainable owing to the age of the patient. Traits which were less frequently described but have been seen in several patients include epicanthic folds, dysplastic ears, and dolichocephaly. ${ }^{79}$

We describe two additional 22q telomere deletions which were unexpectedly detected by FISH after routine cytogenetics failed to discover any visible abnormalities. While both patients have phenotypic similarities to previously described $22 \mathrm{q}$ deletion patients, they were referred for cytogenetic analysis and FISH studies to rule out DiGeorge and Angelman syndromes, respectively. This is the first report of serendipitously detected 22q13.3 deletions following normal cytogenetics results.

\section{Subjects and methods}

CASE REPORTS

Case 1 , aged 5 , is the second child of healthy, non-consanguineous parents. Her older brother is not reported to have any medical problems. The pregnancy was complicated by gestational diabetes, and she was delivered at term by caesarean section because of fetal distress, weighing $2580 \mathrm{~g}$. She required nasogastric feeding for three days postnatally and was subsequently discharged at 5 days of age. Hypotonia and developmental delay were noted in infancy. She did not start walking until $\sim 18$ months and her gait remained unsteady with frequent falls. Her parents also noted a high pain tolerance. The family history included two maternal uncles with dyslexia and one with visual-spatial perception problems.

At 18 months, case 1 was assessed by a neurologist and investigations, including cranial MRI and CT scans as well as brainstem auditory evoked responses (BAERs), were normal. She never had seizures and an EEG at the age of 3 was normal. By the age of $4 \frac{1}{2}$, her persistent generalised developmental delay, particularly in the area of expressive speech, and her unsteady gait raised the possible diagnosis of Angelman syndrome. She was referred for cytogenetic and molecular genetic evaluation.

On examination at 4 years 10 months, her weight $(17.8 \mathrm{~kg})$, height $(112 \mathrm{~cm})$, and head 
circumference $(50.5 \mathrm{~cm})$ were all on the 50 th centile. She had marked hypotonia, lax joints, and an uncoordinated gait. Her facial features were not dysmorphic. Her fingers were relatively wide with broad tips and she had bilateral fifth finger clinodactyly; there was mild 2-3 syndactyly of her toes. Physical examination was otherwise unremarkable. Psychological assessment at the time (Mullen Scales of Early Learning) placed her verbal and non-verbal skills at the 8 to 9 month and the 16 to 17 month level, respectively. The Bayley Scales of Infant Development indicated a mental age equivalent of 11-12 months.

Case 2, aged 5, is the second child of non-consanguineous parents. He was born at term, after an uncomplicated pregnancy, weighing $3742 \mathrm{~g}$ (90th centile). Although his neonatal period was unremarkable, developmental delay was noted in infancy. He sat unassisted at about 9-10 months and walked at 22 months. BAERs were borderline and a cranial MRI scan at the age of $2 \frac{1}{2}$ showed diminished periventricular white matter. Family history included a maternal great aunt with mental retardation and a maternal uncle with learning disabilities.

By the age of 5, case 2 had no speech, but was able to use a few signs. On physical exam-
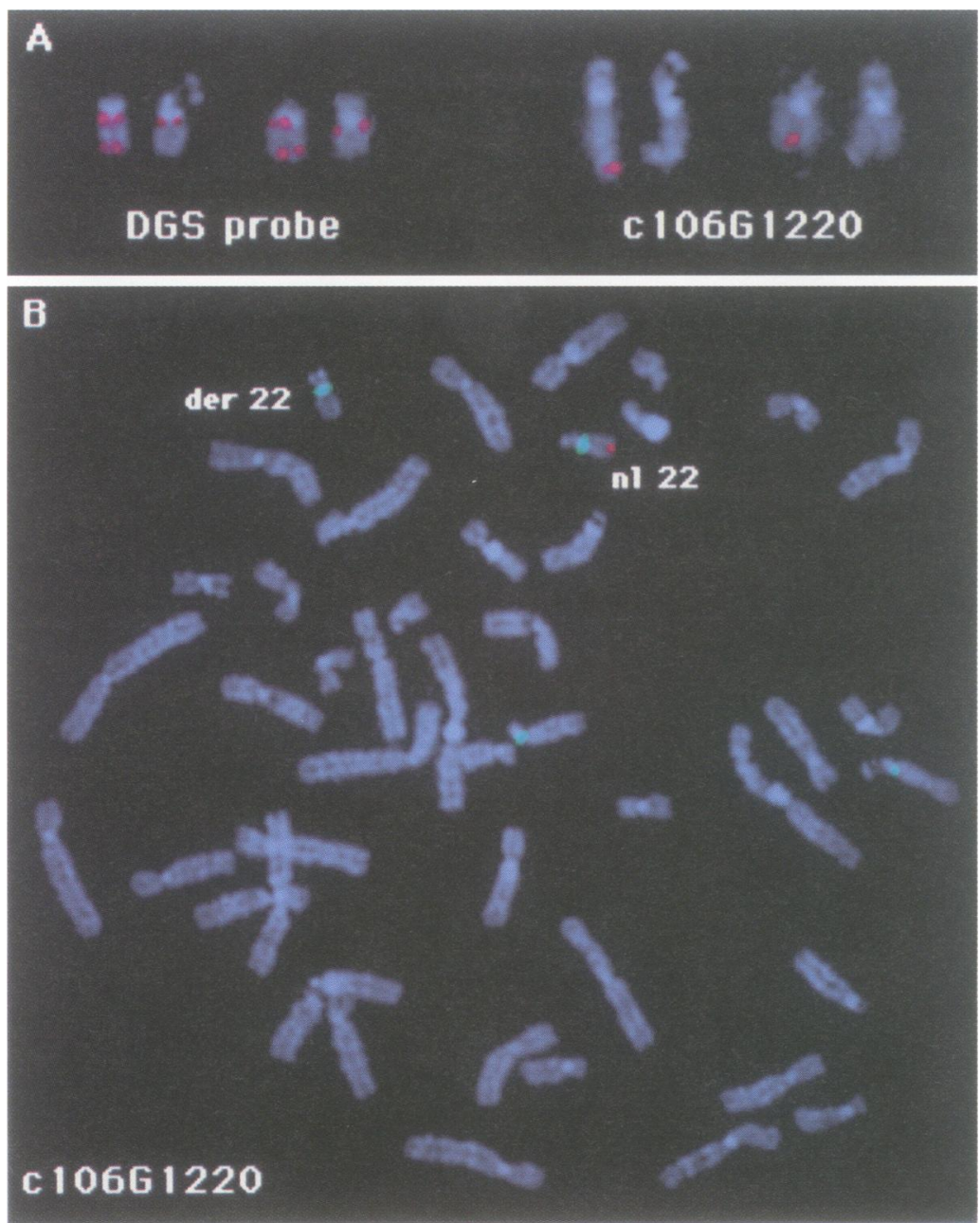

Figure 1 (A) Representative chromosome 22 homologues from FISH analyses on proband 1 using the DGS/VCFS probe (left) and chromosome 22 unique telomere probe c106G1220 (right). Note the deletion of the telomeric probe on each of the right homologues. (B) $A$ metaphase cell from FISH analysis on proband 2 showing a deletion of the chromosome 22 telomere specific probe, c106G1220 (red). Chromosome 22 is identified by a chromosome $14 / 22$ alpha satellite probe (green). ination his height was $107 \mathrm{~cm}$ (25th centile), his weight was $40.5 \mathrm{~kg}$ (50th centile), and his head circumference was $52.5 \mathrm{~cm}$ (75th cen- $\frac{}{2}$ tile). His muscle tone was generally decreased and he made poor eye contact. He had bilateral epicanthic folds, narrow palpebral fissures, and $\stackrel{\Phi}{\frac{}{3}}$ a broad nasal root.

Previous investigations, which were all normal, included peripheral blood cytogenetic $\stackrel{5}{\rightarrow}$ studies, molecular studies for fragile $\mathrm{X}$, serum and urine amino acids, thyroid function tests, $\frac{\bar{\sigma}}{\bar{D}}$ and an ophthalmological examination. He had $\vec{\sigma}_{\bar{\sigma}}$ narrow palpebral fissures, a broad nasal root, and his facial features were somewhat reminis- $\omega$ cent of velocardiofacial syndrome. He was $\vec{\circ}$ referred for further FISH evaluation to rule out $\overrightarrow{-}$ a deletion at $22 \mathrm{q} 11.2$.

\section{CYTOGENETICS AND FISH}

Routine cytogenetic analysis at a greater than w 550 band level was performed on peripheral $\vec{A}$ blood samples from both probands and the parents of case 2 at either the University of Chi- 0 cago Genetic Services (Chicago, IL) or Kaiser Permanente (Oakland, CA) using standard $\vec{z}$ procedures.

Chromosome preparations from peripheral blood were used for fluorescence in situ hybridisation (FISH) analysis. FISH was initially performed on case 1 using D15S10, a probe for $\vec{\theta}$ the Prader-Willi/Angelman region on chromo- $\infty$ some 15 (Oncor Inc, Gaithersburg, MD). Samples from both patients were hybridised with a digoxigenin labelled commercial DiGeorge chromosome region probe, D22S75, containing the telomeric control locus D22S39, according $\mathbb{Q}$ to the manufacturer's conditions (Oncor Inc). The rapid wash procedure was followed with no을 amplification. The two probands were further $\vec{T}$ studied with a chromosome 22q unique tel- 0 omere probe, ICRFc106G1220, labelled with digoxigenin-11-dUTP. ${ }^{11}$ A chromosome 14/220ิ alpha satellite probe, labelled with biotin-16- $\dot{0}$ dUTP, was used to identify chromosome $22 . \stackrel{ }{.}$ Probe preparation, hybridisation, analysis, and $\delta$ imaging were carried out using previously described methods. ${ }^{12}$ Parental chromosome $\frac{\text { c }}{\triangle}$ FISH studies were performed with the commer- $\rightarrow$ cial DiGeorge probe and cosmid 106G1220 to rule out an inherited event.

MICROSATELLITE ANALYSIS

Microsatellite analysis was performed as previ- $\omega$ ously described ${ }^{13}$ to confirm FISH results and determine the parental origin of deletion. $\frac{\mathscr{\Phi}}{\mathscr{D}}$ Whole cell lysates were made from $200 \mu \mathrm{l}$ of $\stackrel{\mathcal{C}}{?}$ peripheral blood using the Qiamp blood lysis 0 kit (Qiagen Inc, Chatsworth, CA). One $\mu$ l of a 1:5 dilution of the cell lysate was used per PCR assay. Five Genethon STR markers from the $\frac{\mathbb{Q}}{\square}$ distal portion of 22q were used: D22S282, S1179, S1140, S928, and S1 169 (markers are listed in order from centromere to telomere).

\section{Results}

CASE 1

Cytogenetic and FISH analyses for Angelman syndrome were requested because of her early hypotonia and persistent lack of speech and 


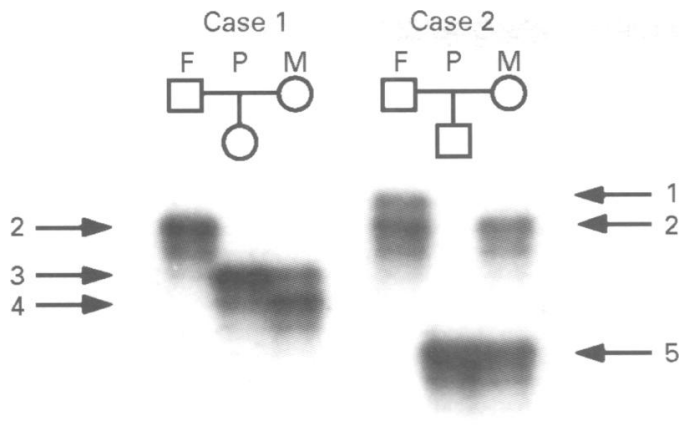

D22S1169

Figure 2 Microsatellite analysis of marker D22S1169 on chromosome 22q13.3 in both probands. In case 1, the father is homozygous for allele 2 , the mother is heterozygous for alleles 3 and 4, and the patient shows inheritance only of maternal allele 3. ( $A$ faint shadow band the same size as allele 4 can be seen in the patient.) In case 2, the father is heterozygous for alleles 1 and 2, the mother is heterozygous for alleles 2 and 5, and the patient inherited only allele 5 from the mother. For both cases the deletion is therefore paternal in origin.

unsteady gait. She was initially determined to have a normal female $(46, \mathrm{XX})$ karyotype, and FISH showed no deletion for the Prader-Willi/ Angelman probe (D15S10) in proximal 15q.

Subsequently, her sample was used alongside that of another patient as a hybridisation control for the DGS/VCFS probe. Surprisingly, a deletion for the telomeric internal control locus (D22S39) was observed on one of her chromosome 22 homologues (fig 1A). This result was confirmed by a chromosome $22 \mathrm{q}$ unique telomere probe (c106G1220), classifying the deletion as terminal, rather than interstitial. Parental FISH studies were normal.

Of five microsatellite markers tested, four markers (D22S282, S1179, S1140, and S928) showed no evidence of a deletion. The most distal marker (D22S1169) was informative for the presence of a maternal band but absence of a paternal allele (fig 2 ), indicating a deletion of paternal origin.

CASE 2

Case 2 was referred for routine cytogenetic and FISH studies to rule out a VCFS deletion. Routine $G$ banding analysis failed to identify any deletion of 22q. While the proximal 22q VCFS locus (D22S575) was present on both homologues, the distal control locus (D22S39) was found to be deleted on one homologue in all cells. Telomere FISH studies were performed using a unique sequence probe (c106G1220) which was also deleted, confirming the initial findings and ruling out an interstitial deletion (fig 1B). Parental chromosome and FISH studies were normal.

Three microsatellite markers (D22S282, S1140, S928) showed the presence of two alleles. The proband showed only one maternal and no paternal allele for the most distal marker (D22S1169), confirming a deletion and indicating a paternal origin (fig 2).

\section{Discussion}

Terminal 22q deletion patients share a common phenotype including hypotonia, developmental delay, and absence of speech. The additional clinical features, which vary among patients, may be the result of the different sizes and mechanisms of the deletions. Further molecular characterisation of all deletions, such as reported by Nesslinger et al, ${ }^{7}$ would be useful for making genotype/phenotype correlations. In light of the initial findings from cytogenetic and microsatellite analyses of our patients, we would expect the deletions to be smaller than those previously reported. Further studies are under way to delineate the size of the deletions in these two cases. A smaller deletion may account for the milder phenotypes present in case 2 , and particularly in case 1. A previously described patient with the smallest molecularly characterised $22 \mathrm{q}$ deletion of $130 \mathrm{~kb}$ showed only mild mental retardation, expressive speech delay, and no other abnormal physical findings. ${ }^{10}{ }^{14}$ Since all of the patients with $22 \mathrm{q}$ terminal deletions are reported to have marked delay or absence of speech, this suggests that a gene(s) involved in expressive speech maps within $130 \mathrm{~kb}$ of the telomere.

While both of our probands share some of the clinical features of previously reported $22 \mathrm{q}$ telomere deletions, they were initially referred for cytogenetic studies for two different syndromes. Hypotonia and developmental delay are features commonly observed in many syndromes; however, in association with severe speech delay, these phenotypes may be a significant indication to test for a $22 \mathrm{q}$ deletion. The phenotypic similarity of these deletion patients to Angelman syndrome is particularly noteworthy, as it raises the possibility of a $22 \mathrm{q}$ deletion subset in patients with Angelman-like features in whom the syndrome has been ruled out. This hypothesis could be tested by combining $22 \mathrm{q}$ telomere FISH analysis with Angelman studies to determine if the overall deletion detection rate in such patients is increased.

The de novo deletion in both of our probands was determined to be of paternal origin. Two previously reported cases ${ }^{6}{ }^{10}$ were also paternal deletions. However, in four further cases, ${ }^{17910}$ a maternal origin of the deletion was reported. Therefore, imprinting is an unlikely mechanism associated with the phenotype in these deletions.

The recently increasing number of reported $22 \mathrm{q}$ telomere deletions indicates that these types of deletion may be occurring more frequently than previously thought. The chance discovery of our two cases by FISH shows that detection of smaller deletions by routine cytogenetics is difficult because of its limited sensitivity and because the $22 \mathrm{q}$ telomeric region is $G$ negative. Routine cytogenetic analysis or the use of whole chromosome painting probes would therefore be expected to miss smaller 22q telomere rearrangements. We conclude that FISH studies using telomeric probes for 22q are most suitable for ruling out and confirming deletions of this region.

We would like to acknowledge Dr Daniela Pilz for her assistance in manuscript preparation, Patti L Mills, MS and Christine Hartlove, MS for patient/sample coordination, and the University of Chicago Genetic Services laboratories for cell culture and sample preparation. 
1 Watt JL, Olson IA, Johnston AW, Ross HS, Couzin DA Stephen GS. A familial pericentric inversion of chromosome 22 with a recombinant subject illustrating a 'pure' pome 22 with a recombinant subject illustrating a 'pure'

2 Romain DR, Goldsmith J, Cairney H, Columbano-Green LM, Smythe RH, Parfitt RG. Partial monosomy fo chromosome 22 in a patient with $\operatorname{del}(22)$ (pter $\rightarrow$ q13.1: q13.33 $\rightarrow$ qter). F Med Genet 1990;27:588-9.

3 Herman GE, Greenberg F, Ledbetter DH. Multiple congenital anomaly/mental retardation (MCA/MR) syndrome with Goldenhar complex due to a terminal del(22q). Am f Med Genet 1988;29:909-15.

4 Zwaigenbaum L, Siegel-Bartelt J, Teshima I, Ho C. Two patients with 22q13.3 deletions have similar facies and patients with 22q13.3 deletions have similar facies and
developmental patterns. Am $\mathcal{f}$ Hum Genet 1990;47:A45.

5 Narahara K, Takahashi Y, Murakami M, et al. Terminal 22 deletion associated with a partial deficiency of arylsulphatase A. F Med Genet 1992;29:432-3.

6 Phelan MC, Thomas GR, Saul RA, et al. Cytogenetic, biochemical, and molecular analyses of a 22q13 deletion. $\mathrm{Am}$ ¥ Med Genet 1992;43:872-6.

7 Nesslinger NJ, Gorski JL, Kurczynski TW, et al. Clinical, cytogenetic, and molecular characterization of seven patients with deletions of chromosome 22q13.3. Am $\mathcal{F} \mathrm{Hum}$ Genet 1994;54:464-72.
8 Yong YP, Knight LA, Yong MH, Lam S, Ho LY. Partial monosomy for chromosome 22 in a girl with mental retarmonosomy for chromosome 22 in a girl
dation. Singapore Med $₹ 1997 ; 38: 85-6$.

9 Doheny KF, McDermid HE, Harum K, Thomas GH, Ray-C mond GV. Cryptic terminal rearrangement of chromosome 22q13.32 detected by FISH in two unrelated patients. Fळ
Med Genet 1997;34:640-4.

10 Flint J, Wilkie AO, Buckle VJ, Winter RM, Holland AJ, McDermid HE. The detection of subtelomeric chromo-D somal rearrangements in idiopathic mental retardation. Nat Genet 1995;9:132-40.

11 Ning $\mathrm{Y}$, Rosenberg $M$, Biesecker LG, Ledbetter $\mathrm{DH}$. Isolation of the human chromosome 22q telomere and its appli- $\overline{\vec{F}}$ cation to detection of cryptic chromosomal abnormalities Hum Genet 1996;97:765-9.

12 Chong SS, Pack SD, Roschke AV, et al. A revision of the lis-드 sencephaly and Miller-Dieker syndrome critical region in chromosome 17p13.3. Hum Mol Genet 1997;6:147-55.

13 Christian SL, Robinson WP, Huang B, et al. Molecular characterization of two proximal deletion breakpoints in both Prader-Willi and Angelman syndrome patients. Am fô Hum Genet 1995;57:40-8.

14 Wong AC, Ning Y, Flint J, et al. Molecular characterization $\vec{\circ}$ of a $130-\mathrm{kb}$ terminal microdeletion at $22 \mathrm{q}$ in a child withmild mental retardation. Am $\mathcal{F}$ Hum Genet 1997;60:113-20. $\vec{\omega}$ 